\title{
Research and Implementation of Wireless Personal Area Network based on IEEE802.15.4
}

\author{
Liugang Zheng \\ Shanghai Monitoring Station the State Radio_Monitoring_Center, Shanghai 201419 \\ hunter2011@foxmail.com
}

Keywords:802.15.4, WPAN, WSN, Implementation.

\begin{abstract}
IEEE 802.11 wireless LAN's popularity, and IEEE 802.16 wireless broadband network in the commercial standardization in recent years, have made significant wireless network technology increasingly important, but also more and more applications and products has emerged. While wireless personal area network there since Bluetooth, also gradually begun to be noticeable, and with personal portable digital devices popular (such as cell phones, PDAs), and has accelerated wireless personal area network technology in the study and application of one of the -- wireless sensor networks are also began to be widely discussed on topic.
\end{abstract}

\section{Introduction}

Wireless Sensor Network is used to detect the change of some physical conditions such as the change of temperature, humidity, sound and pressure. And it uses the widely distributed sensor nodes in wireless sensor network to detect specific projects in specific area, and it sends the detected data to the specific Sink by wireless transmission technique to make the succeeding data process and analysis, and makes the corresponding actions. And wireless sensor network is widely applied. For wireless transmission protocol for wireless sensor network, there is no definite requirement. In the relevant researches, only IEEE 802.11 DCF[1] and IEEE 802.11 are modified to meet wireless sensor network. In addition, there are several communication protocols (SMACS, EAR, Hybird TDMA/FDMA) for the application of wireless sensor network.

\section{Development of IEEE 802.15.4 and Introduction of Features}

There are Four work groups in IEEE 802.15, and the difference of them is data transmission rate, power consumption degree and service quality, as shown in Table 1.

Table 1 IEEE 802.15 work group

\begin{tabular}{|c|c|c|c|c|}
\hline Project & Data Rate & Range & configuration & Other Features \\
\hline 802.15 .1 & $1 \mathrm{Mbps}$ & $10 \mathrm{~m}^{\sim} 100 \mathrm{~m}$ & $\begin{array}{c}8 \text { active } \\
\text { device }\end{array}$ & $\begin{array}{c}\text { Authentication, En } \\
\text { cryption, Voice }\end{array}$ \\
\hline 802.15 .3 & $\begin{array}{c}22,33,44,55 \\
\text { Mbps }\end{array}$ & $10 \mathrm{~m}$ & $\begin{array}{c}256 \text { active } \\
\text { device }\end{array}$ & $\begin{array}{c}\text { FCC, Qos, Fast } \\
\text { Join, Multi-Media }\end{array}$ \\
\hline 802.15 .4 & $250 \mathrm{Kbps}$ & $1 \mathrm{~m} \sim 10 \mathrm{~m}$ & $\begin{array}{c}\text { Master/Slave, } \\
\text { peer to peer }\end{array}$ & Battery life \\
\hline 802.15 .2 & $\begin{array}{c}\text { Coexistence } \\
\text { Model and } \\
\text { Mechanism }\end{array}$ & & & \\
\hline
\end{tabular}

IEEE 802.15.4 is a new communication protocol. Short-distance and low -transmission rate physical layer and Medium access control layerwas initially defined in Draft 18 in February 2003.

The features of IEEE 802.15.4 LR-WPAN are as follows.

(1) Operating frequency band has three choices.

In $2.4 \mathrm{GHz}$ ISM frequency band, there are 16 channels and the transmission rate is $250 \mathrm{Kbps}$.

In $915 \mathrm{MHz}$ ISM frequency band, there are 10 channels and the transmission rate is $40 \mathrm{Kbps}$.

In European $868 \mathrm{MHz}$ frequency band, there is 1 channel, and the transmission rate is $20 \mathrm{Kbps}$.

(2) Star or Peer to Peer topology can be used. And it can support Cluster Tree framework in the future. 
(3) It supports low latency device.

(4) It uses IEEE 802.11 CSMA/CA for communication.

(5) It supports 64-bit extended address and 16-bit short address.

(6) Acknowledgment in data link layer can be optionally used.

(7) It uses low-energy consumption and has short duty-cycle $<0.1 \%$.

The system obeying IEEE802.15.4 standard consists of several parts. The primary part is device. A device may be full-function device or reduced-function device. Two or more devices can compose a WPAN for communication by sharing channels in personal operation space, but there is a FFD at least in a network, which is used as PAN coordinator.

IEEE 802.15.4 can used different WPAN topological structures according to the application requirements, and IEEE 802.15.4 supports start (in Figure 1) and peer-to-peer topological structure ( in Figure 2).

In star structure, a central controller (PAN coordinator) only can connect with other devices for communication, and the device without PAN coordinator can't communicate. PAN coordinator is the control manager of PAN. The devices which operate on any topological network need to have the unique 64-bit extended address. The address can be used for direct communication in Pan. Or when the device is connected with PAN coordinator, the extended address can be replaced by a 16-bit short address which is distributed by PAN coordinator.

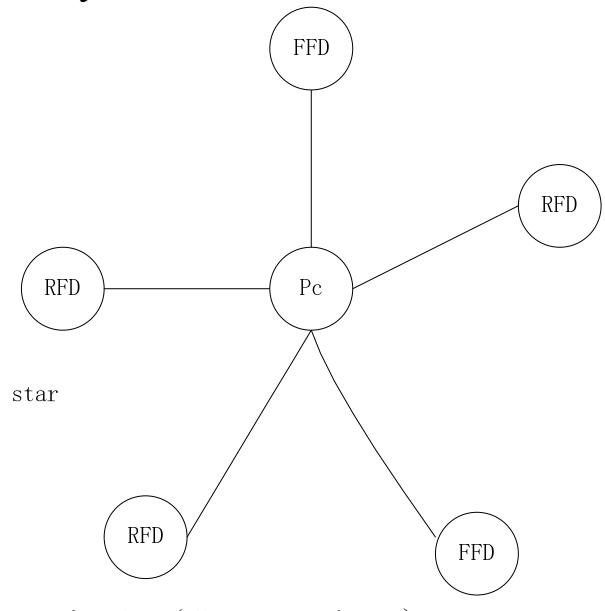

Fig. 1 (Star Topology)

The network with peer-to-peer topology also has a PAN coordinator. The most evident difference from start topology is that any device and communicate in the network. Peer-to-peer topology can compose more complicated network such as Mesh network. A peer-to-peer network may be Ad hoc network which can be self-organizing and self-repairing. And the information can be transmitted in multiple jumping way between devices in the network. 

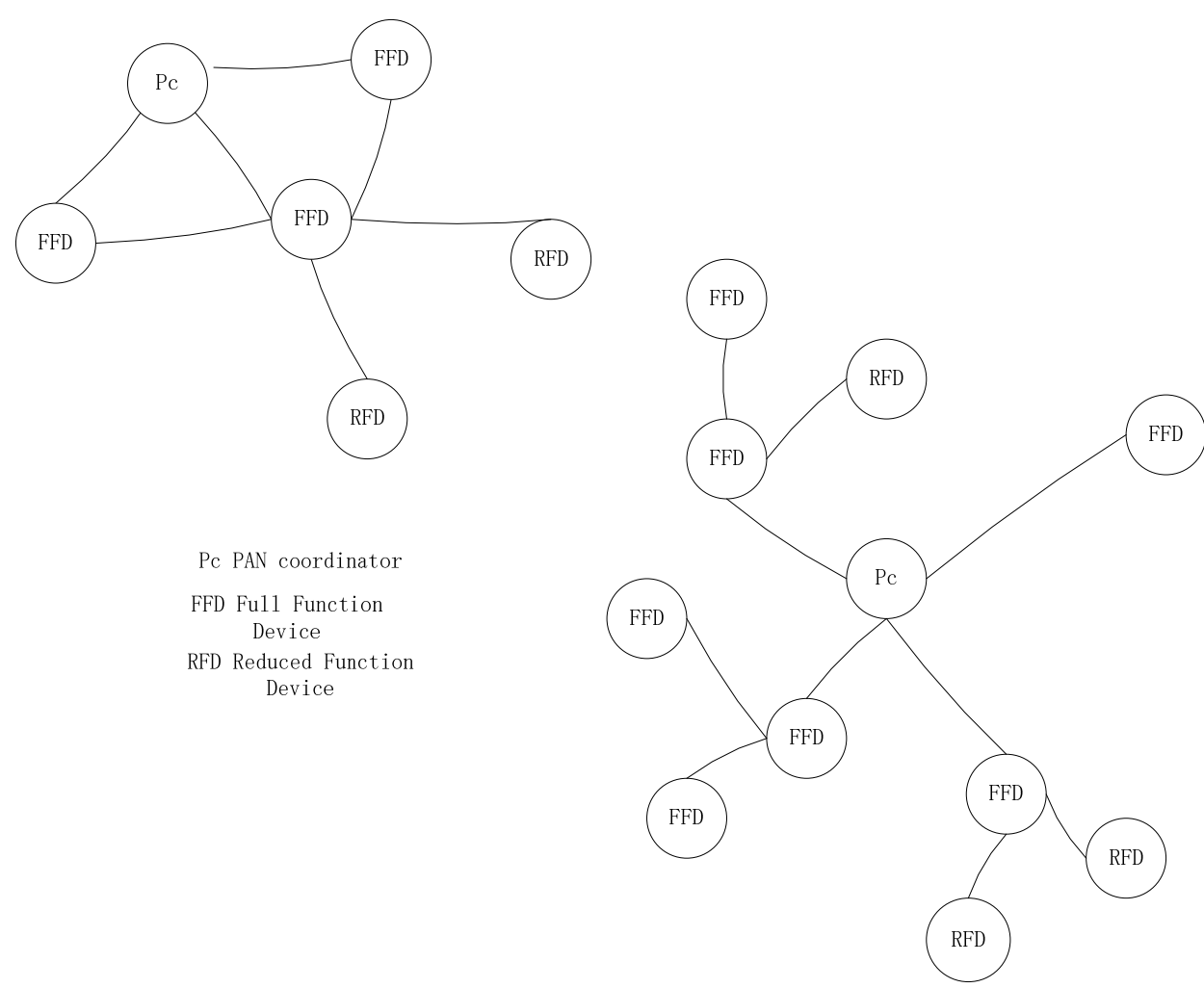

Fig. 2 Mesh and Cluster Tree Topology

IEEE 802.15.4WPAN network has the following characteristics.

In the network, the data transmission providers three rates, $250 \mathrm{kbps}, 40 \mathrm{kbps}$ and $20 \mathrm{kbps}$. Different transmission rates are determined according to the selected frequency band (physical layer).

The network is start or peer-to-peer topological structure.

Each device distributes a 16-bit short address or 64-bit extended address in the network.

It uses CSMA/CA channel access technology.

It uses fully acknowledged protocol to provide reliable transmission.

Low power consumption.

Energy detection.

Link quality indication.

$2.45 \mathrm{GHz}$ frequency band is divided into 16 channels, $915 \mathrm{MHz}$ frequency band is divided into 10 channels, and $868 \mathrm{MHz}$ frequency band is only divided into 1 channel.

\section{Overall Design of Hardware Platform}

In hardware platform, the functions which are implemented by microcontroller and the peripheral modules are as follows.

The control radio frequency module completes data reception and transmission.

The control sensor module makes data collection.

The host receiver controls and completes data exchange.

The microcontroller is the central module, and is communicated by SPI bus or I/O ports controlling RF module. The data collected by sensor module is connected by SPI bus or microcontroller. And the communication of nodes and PC can be implemented by RS232 serial bus or serial transferring USB interface.

The advantages of JN5121 chip design are as follows.

(1) Single chip solution integrates transceiver and microcontroller, and it is appropriate for wireless sensor network application.

(2) Compared with sensor and controller, it has small volume and low power consumption. 
(3) The design is compacted, and the occupied space is small, which can reduce PCB size of the users greatly.

(4) MAC address of hardware design reduces the power consumption of system and overload of processor.

(5) It has extended external device interface.

So using standard hardware device developed by Versa based on JN5121 chip is divided into central controller, router and terminal device.

Central controller

It has the characteristics of small volume, wide coverage range and knowing the number of subnodes. The superficial area of the device is about $60 \mathrm{~mm} * 40 \mathrm{~mm}$. It can be external to the antennas with different gains, and the furthest covering radius can achieve 2 kilometers.

Router

In 802.15.4 network, router is the trunk equipment to transmit the data in the network, and the user can use the router to extend the range of network.

Terminal equipment

Terminal equipment and router use the same hardware design. Both of them are equipped with sensor interface and standard serial port. The users can use the devices to monitor sensor data or make other applications.

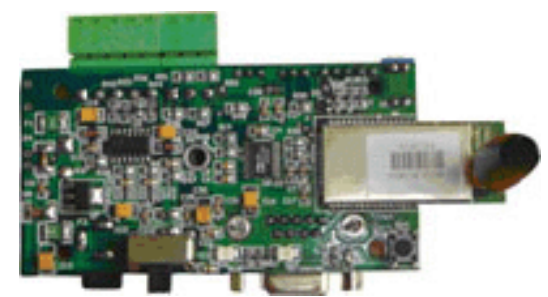

Fig. 3 Physical picture of hardware platform (reverse side)

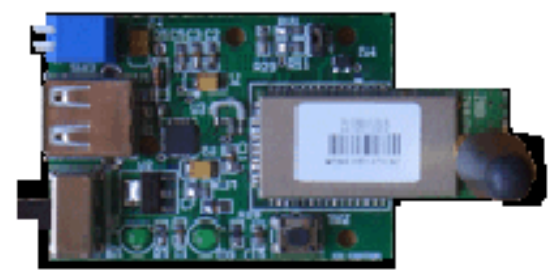

Fig. 4 Physical picture of hardware platform (front side)

Characteristics of the device

(1) Power supply mode is diversified.

Two $7^{\text {th }}$ batteries.

External power source.

Solar power.

(2) Several interface selections

2 analog quantity input interfaces.

2 digital quantity input interfaces.

1 digital quantity output interface.

1 RS232 interface.

\section{System Software Architecture}

Firstly, knowing the protocol stack architecture of IEEE 802.15.4 can refer to the following:

(1) Applications interact by protocol stack API of 802.15.4 and protocol layer of IEEE 802.15.4. The interaction implements the request and confirmation of MCPS/MLME, and the identification and response of message. IEEE 802.15.4 protocol layer interacts with the hardware and register of the lower layer.

(2) Applications interact by integrating external device API and the integrated external devices on chips (such as AD, DA, DIO and Timers...). 
(3) Applications access the devices on board card by sensor board of API and development kits or controller, such as sensor and son on.

(4) Hardware layer generates various interruptions and transfers to each software module by interrupters.

(5) Developers can use the provided application queue API to simplify the process of communication protocol layer and hardware API interruption.

\section{Conclusions}

IEEE 802.15.4 develops rapidly in short-distance wireless personal area network technology with incomparable advantages. And the technique must be the research not of colleges and research institutions in years to come. IEEE 802.15.4 technique has wide application prospect and market value. With the deepening of research, IEEE 80.15.4 technique will be applied in all life areas, which influences and changes the life style of the people, and makes the people live in a modern information network full of vigor.

\section{References}

[1] D. Tian and N. D. Georganas, "A coverage-preserving node scheduling scheme for large wireless sensor networks," in Proceedings of the first ACM international workshop on Wireless sensor networks and applications, pp. 32-41, 2002.

[2] J. Deng, Y. S. Han, B. Heinzelman, and P. K. Varshney, "Scheduling Sleeping Nodes in High Density Cluster-based Sensor Networks," submitted to the IEEE International Conference on Communications, 2004.

[3] J. Deng, Y. S. Han, B. Heinzelman, and P. K. Varshney, "Balanced-energy sleep scheduling scheme for high density cluster-based sensor networks," submitted to the Third International Symposium on Information Processing in Sensor Networks (IPSN’04), 2004.

[4] K. Sohrabi and G. J. Pottie, "Performance of a novel self-organization protocol for wireless ad hoc sensor network," in Proceedings of the IEEE 50th Vehicular Technology Conference, pp. 1222-1226,1999.

[5] W. Ye, J. Heidemann, D. Estrin, "An energy-efficient MAC protocol for wireless sensor networks," IEEE INFOCOM 2002, pp. 1567-1576, 2002. 